\title{
CONGRUENCES AND IDEALS ON BOOLEAN MODULES: A HETEROGENEOUS POINT OF VIEW
}

\author{
SANDRA MARQUES PINTO AND M. TERESA OLIVEIRA-MARTINS
}

\begin{abstract}
Definitions for heterogeneous congruences and heterogeneous ideals on a Boolean module $\mathcal{M}$ are given and the respective lattices $\operatorname{Cong} \mathcal{M}$ and $I d e \mathcal{M}$ are presented. A characterization of the simple Boolean modules is achieved differing from that given by Brink in a homogeneous approach. We construct the smallest and the greatest modular congruence having the same Boolean part. The same is established for modular ideals. The notions of kernel of a modular congruence and the congruence induced by a modular ideal are introduced to describe an isomorphism between $\operatorname{Cong} \mathcal{M}$ and Ide $\mathcal{M}$. This isomorphism leads us to conclude that the class of the Boolean module is ideal determined.
\end{abstract}

KEYWORDS: Relation algebras; Boolean modules; modular heterogeneous congruence; modular heterogeneous ideal; simple Boolean module.

AMS Subject Classification (2010): 03B70, 03G05, 03G15, 06B10, 06E25, 08A68.

\section{Introduction}

The application of abstract algebra in logic and computer science rely and depends on the simultaneous study of algebras of sets and algebras of binary relations. To talk about algebras of sets is synonymous to study Boolean algebras and the most famous algebra of relations is that presented by Tarski in [9]. There Tarski introduces the relations algebras as algebras of binary relations adding to the Boolean structure the operations of composition, converse and identity. Boolean modules were first established by Brink in [1]. Given a relation algebra $\mathcal{R}$, Brink defined and studied Boolean $\mathcal{R}$-modules as a Boolean algebra $\mathcal{B}$ with actions from the relation algebra $\mathcal{R}$. Such actions were induced by a map called Peircean operator, :, from $\mathcal{R} \times \mathcal{B}$ to $\mathcal{B}$, where each element $a \in \mathcal{R}$ defines in $\mathcal{B}$ a map

$$
\begin{aligned}
\mathcal{B} & \rightarrow \mathcal{B} \\
p & \mapsto a: p
\end{aligned}
$$

Received July 29, 2010.

Financial support from the CMUC, Department of Mathematics, University of Coimbra, 3001454 Coimbra, Portugal, is gratefully acknowledged. 
satisfying a required set of axioms. A unified concept associated to this homogeneous approach is given naturally by a two sorted algebra $\mathcal{M}=(\mathcal{B}, \mathcal{R},:)$ containing a Boolean algebra $\mathcal{B}$ and a relation algebra $\mathcal{R}$ where the Peircean operator, :, is interpreted now as a heterogeneous operation in $\mathcal{M}$ ranging from $\mathcal{R} \times \mathcal{B}$ to $\mathcal{B}$. The importance of the heterogeneous algebras approach on Boolean modules is fully presented on the introduction of [6] by R. Hirsch and [2] by Brink, Britz and Schmidt. Nevertheless, their characterization of simple Boolean modules follows a homogeneous point of view, since their definition of a Boolean module ideal is a Boolean algebra ideal closed under multiplication by elements of the relation algebra. The same can be stated concerning congruences. A throughout heterogeneous approach is followed in our work for both concepts under study (Definitions 3.1 and 4.1). Thus a modular congruence $\theta$ is considered as adequate pair of congruences $\theta=\left(\theta_{1}, \theta_{2}\right)$ with $\theta_{1}$ a Boolean congruence and $\theta_{2}$ a relation congruence and modular ideals $I$ as suitable pairs of ideals $I=\left(I_{1}, I_{2}\right)$ where $I_{1}$ is a Boolean ideal and $I_{2}$ is a ideal on the relation algebra.

\section{Boolean modules}

Boolean modules were introduced by Brink [1] as homogeneous algebras, Boolean algebras with a multiplication (Peircean product) from a relation algebra. A Boolean module is, from a heterogeneous point of view, a two sorted algebra containing a Boolean algebra, a relation algebra and an operator (a heterogeneous operation, the Peircean operator) taking a pair of a relation algebra element and a Boolean algebra element and originating a Boolean algebra element. We present here the standard definition of relation algebras given by Brink (originated from Chin and Tarski [3] and modified in Tarski [10]).

Definition 2.1. A relation algebra is an algebra $\mathcal{R}=\left(R, \vee, \wedge,{ }^{\prime}, \mathrm{o}, 1, ;,{ }^{\prime}, e\right)$ satisfying the following axioms for each $a, b, c \in R$

$\mathrm{R} 1 \quad\left(R, \vee, \wedge,{ }^{\prime}, \mathrm{o}, 1\right)$ is a Boolean algebra

R2 $a ;(b ; c)=(a ; b) ; c$

R3 $a ; e=a=e ; a$

R4 $a^{u}=a$

R5 $\quad(a \vee b) ; c=a ; c \vee b ; c$

R6 $\quad(a \vee b)^{\triangleleft}=a \vee \vee b$

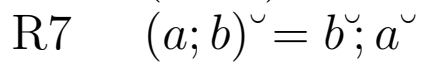

R8 $\quad a^{\prime} ;(a ; b)^{\prime} \leq b^{\prime}$. 
Notation. For $a, b \in R$ we also write $a b$ instead of $a ; b$.

As usual, for elements $p, q$ on a Boolean algebra $B$ we define $p \oplus q=$ $\left(p \wedge q^{\prime}\right) \vee\left(p^{\prime} \wedge q\right)$. In particular, for elements $a, b$ on a relation algebra $R$ we also define $a \oplus b=\left(a \wedge b^{\prime}\right) \vee\left(a^{\prime} \wedge b\right)$.

The standard class of models of relation algebras is the class of proper relation algebras.

Definition 2.2. A proper relation algebra over a non-empty set $U$ is a set of binary relations on $U$ that contains the identity relation and is closed with respect to union, intersection, complementation, relational composition and converse. If a proper relation algebra consists of all binary relations defined on $U$, then this algebra is called the full relation algebra and is denoted by $\mathcal{R}(U)$. More precisely, $\mathcal{R}(U)$ is the power set algebra over $U^{2}$ endowed with composition (";"), converse ("v") and identity ("Id") operations defined, for $a, b \subseteq U^{2}$, by

$a ; b=\{(s, t):$ exists $u \in U$ such that $(s, u) \in a$ and $(u, t) \in b\}$

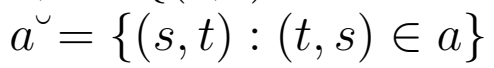

$I d=\{(s, s): s \in U\}$.

The arithmetic of relation algebras can be described by the facts assembled on the following theorem.

Theorem 2.3. On any relation algebra $\mathcal{R}$ the following hold for any $a, b, c, d \in$ $R$

$R 9 \quad \breve{e}=e, \quad \breve{o}=o, \quad \breve{1}=1$

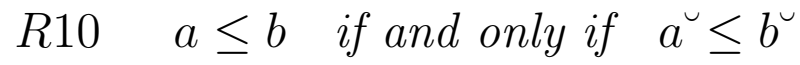

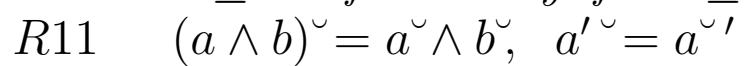

$R 12 \quad a ; o=o=o ; a, 1 ; 1=1$

$R 13 \quad a(b \vee c)=a b \vee a c$

$R 14 \quad$ If $a \leq b$ then $c a \leq c b$ and $a c \leq b c$.

$R 15 \quad(a b) \wedge c=o$ if and only if $(\overrightarrow{a c}) \wedge b=o$ if and only if $(c b) \wedge a=o$

$R 16 \quad(a b) \wedge(c d) \leq a\left[(\overrightarrow{a c}) \wedge\left(b d^{y}\right)\right] d$

$R 17 \quad(a \oplus b)^{\iota}=a^{\iota} \oplus b^{\iota}$.

Proof: R9-R16 are proved in [3]. To prove R17 we use R6 and R11. Thus

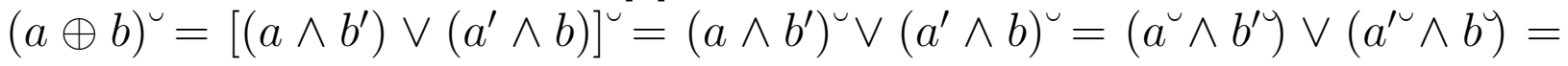

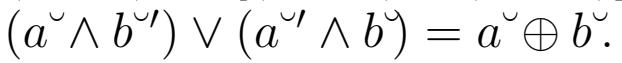

As mentioned before, Brink introduced the notion of a Boolean $\mathcal{R}$-module $\mathcal{B}$ as a homogeneous algebra. Here, the heterogeneous approach followed in 
this study is emphasized from the very beginning, on the following definition, where the roles of $\mathcal{B}$ and $\mathcal{R}$ are taken evenly.

Definition 2.4. A Boolean module is a two-sorted algebra $\mathcal{M}=(\mathcal{B}, \mathcal{R},:)$ where $\mathcal{B}$ is a Boolean algebra, $\mathcal{R}$ is a relation algebra and : is a mapping $\mathcal{R} \times \mathcal{B} \longrightarrow \mathcal{B}$ (written $a: p$ ) such that for any $a, b \in R$ and $p, q \in B$, the following assertions are satisfied.

M1 $\quad a:(p \vee q)=a: p \vee a: q$

M2 $\quad(a \vee b): p=a: p \vee b: p$

M3 $\quad a:(b: p)=(a ; b): p$

M4 $e: p=p$

M5 o: $p=0$

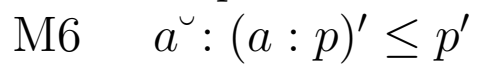

Notation. For $a, b \in R$ and $p \in B$ we also use ap to represent $a: p$.

The standard models of Boolean modules are provided by the class of proper Boolean modules.

Definition 2.5. A proper Boolean module is a two-sorted algebra of a proper Boolean algebra (a field of sets) and a proper relation algebra together with Peirce product defined on sets and relations. For any relation $a$ over some non-empty set $U$ and any subset $p$ of $U$, the Peirce product : of $a$ and $p$ is defined by

$$
a: p=\{s \in U: \text { there exists } t \in p \text { such that }(s, t) \in a\} .
$$

A full Boolean module $\mathcal{M}(U)$ over a non-empty set $U$ is the Boolean module $(\mathcal{B}(U), \mathcal{R}(U),:)$, where $\mathcal{B}(U)$ is the power set algebra over $U, \mathcal{R}(U)$ is the full relation algebra over $U$ and : is the Peirce product defined set-theoretically.

Some facts valid on Boolean modules deserve mention.

Theorem 2.6. On any Boolean module $\mathcal{M}=(\mathcal{B}, \mathcal{R},:)$ the following hold for any $a, b \in R$ and $p, q \in B$

$M 7$ If $p \leq q$ then $a p \leq a q$.

M8 If $a \leq b$ then $a p \leq b p$.

M9 $a(p \wedge q) \leq(a p \wedge a q)$

$M 10 \quad(a \wedge b) p \leq(a p \wedge b p)$

M11 ap $\wedge q=0$ if and only if $a \breve{q} \wedge p=0$

$M 12$ If $\sum_{i \in I} p_{i}$ exists, then so does $\sum_{i \in I} a p_{i}$, and $a \sum_{i \in I} p_{i}=\sum_{i \in I} a p_{i}$.

$M 13 a 0=0$ 
$M 14 \quad 1: 1=1$

$M 15 \quad(a 1)^{\prime} \leq a^{\prime} 1$

$M 16 \quad$ ap $\wedge q \leq a(p \wedge a \breve{q})$

$M 17 p \leq 1 p$

Proof: Proved in [1].

\section{The lattice $C o n g \mathcal{M}$ of modular congruences}

The concept of congruence with its recognized unifier formulation plays a central role both on lattice and universal algebra theories in general. Once more the presentation of next notion follows a heterogeneous view-point.

Definition 3.1. Let $\mathcal{M}=(\mathcal{B}, \mathcal{R},:)$ be a Boolean module. A pair $\theta=\left(\theta_{1}, \theta_{2}\right)$ is a (modular) congruence relation on $\mathcal{M}$ if $\theta_{1}$ is a congruence relation on $\mathcal{B}$, $\theta_{2}$ is a congruence relation on $\mathcal{R}$ and $a p \theta_{1} b q$ whenever $\left(p \theta_{1} q\right.$ and $\left.a \theta_{2} b\right)$.

Let us denote by $\operatorname{Cong} \mathcal{M}$ the set of all modular congruences defined on a Boolean module $\mathcal{M}$. The set $\operatorname{Cong} \mathcal{M}$ is partially ordered by $\left(\theta_{1}, \theta_{2}\right) \leq$ $\left(\gamma_{1}, \gamma_{2}\right)$ if and only if $\theta_{1} \subseteq \gamma_{1}$ and $\theta_{2} \subseteq \gamma_{2}$. Our next aim is to define the lattice structure $\left(\operatorname{Cong} \mathcal{M}, \wedge_{\mathcal{M}}, \vee_{\mathcal{M}}\right)$. Since the intersection $\theta \cap \gamma=\left(\theta_{1} \cap \gamma_{1}, \theta_{2} \cap \gamma_{2}\right)$ of any two modular congruences $\theta$ and $\gamma$ defined on $\mathcal{M}$ is, itself, a modular congruence on $\mathcal{M}$, let $\theta \wedge_{\mathcal{M}} \gamma=\theta \cap \gamma$. Let us use $\langle\theta\rangle_{\mathcal{A}}$ to represent the congruence relation generated by the binary relation $\theta$ on any (homogeneous or heterogeneous) algebra $\mathcal{A}$, i.e., the intersection of all congruence relations $\theta^{\prime}$ on $\mathcal{A}$ containing $\theta$

$$
\langle\theta\rangle_{\mathcal{A}}=\cap\left\{\theta^{\prime}: \theta^{\prime} \in C o n g \mathcal{A} \text { and } \theta \subseteq \theta^{\prime}\right\} .
$$

Now we need to define $\theta \vee_{\mathcal{M}} \gamma=\left(\tau_{1}, \tau_{2}\right)$. Using the classic definition of supremum of two congruences, the relation part of the congruence $\theta \vee_{\mathcal{M}} \gamma$ can be given by $\tau_{2}=\theta_{2} \vee_{\mathcal{R}} \gamma_{2}=\left\langle\theta_{2} \cup \gamma_{2}\right\rangle_{\mathcal{R}}$. As far as the Boolean part is concerned some caution is required. Since the Boolean part must be closed to the operation : evolving elements of $R$, we could be led to think of enlarging $\left\langle\theta_{1} \cup \gamma_{1}\right\rangle_{\mathcal{B}}$ with, for instance, elements of the type $(a p, b q)$ with $(a, b) \in \theta_{2}$ and $(p, q) \in \gamma_{1}$. In fact, that is not necessary, as shown below.

Proposition 3.2. Let $\mathcal{M}=(\mathcal{B}, \mathcal{R},:)$ be a Boolean module. For $\theta, \gamma \in$ $\operatorname{Cong} \mathcal{M},(p, q) \in\left\langle\theta_{1} \cup \gamma_{1}\right\rangle_{\mathcal{B}}$ and $(a, b) \in\left\langle\theta_{2} \cup \gamma_{2}\right\rangle_{\mathcal{R}}$ we have $(a p, b q) \in\left\langle\theta \theta_{1} \cup \gamma_{1}\right\rangle_{\mathcal{B}}$.

Proof: Analogous to proposition on dynamic algebra [8]. 
The structure $\left(\operatorname{Cong} \mathcal{M}, \wedge_{\mathcal{M}}, \vee_{\mathcal{M}}\right)$ where, for every $\theta, \gamma \in C o n g \mathcal{D}$ the operations are defined by

$$
\begin{aligned}
& \theta \wedge_{\mathcal{M}} \gamma=\theta \cap \gamma=\left(\theta_{1} \cap \gamma_{1}, \theta_{2} \cap \gamma_{2}\right) \\
& \theta \vee_{\mathcal{M}} \gamma=\langle\theta \cup \gamma\rangle_{\mathcal{M}}=\left(\left\langle\theta_{1} \cup \gamma_{1}\right\rangle_{\mathcal{B}},\left\langle\theta_{2} \cup \gamma_{2}\right\rangle_{\mathcal{R}}\right)
\end{aligned}
$$

is a lattice called the congruence lattice $\operatorname{Cong} \mathcal{M}$ of $\mathcal{M}$.

In a Boolean module $\mathcal{M}=(\mathcal{B}, \mathcal{R},:)$ we define congruences $\Delta_{B}$ and $\nabla_{B}$ on $B$ and $\Delta_{R}$ and $\nabla_{R}$ on $R$ as expected

$$
\begin{array}{ll}
\Delta_{B}=\{(p, p): p \in B\}, & \nabla_{B}=\{(p, q): p, q \in B\}, \\
\Delta_{R}=\{(a, a): a \in R\}, & \nabla_{R}=\{(a, b): a, b \in R\} .
\end{array}
$$

One can easily show that the pairs $\left(\Delta_{B}, \Delta_{R}\right),\left(\nabla_{B}, \nabla_{R}\right)$ and $\left(\nabla_{B}, \Delta_{R}\right)$ are congruences on $\mathcal{M}$, but in general $\left(\Delta_{B}, \nabla_{R}\right)$ is not a congruence on $\mathcal{M}$.

Proposition 3.3. On a Boolean module $\mathcal{M}=(\mathcal{B}, \mathcal{R},:)$ the pair $\left(\theta_{1}, \nabla_{R}\right)$ is a modular congruence on $\mathcal{M}$ if and only if $\theta_{1}=\nabla_{B}$.

Proof: If $\left(\theta_{1}, \nabla_{R}\right)$ is a modular congruence on $\mathcal{M}$ then $1 \theta_{1} 1$ and o $\nabla_{R} 1$ and then $\mathrm{o} 1 \theta_{1}(1: 1)$. So $0 \theta_{1} 1$, i.e., $\theta_{1}=\nabla_{B}$.

On an arbitrary Boolean module $\mathcal{M}=(\mathcal{B}, \mathcal{R},:)$ (not full) it is possible that for some relation algebra elements $a$ and $b$ we may have $a p=b p$ for all $p \in B$ without having $a=b$. Boolean modules for which this situation is forbidden is presented next.

Definition 3.4. A Boolean module $\mathcal{M}=(\mathcal{B}, \mathcal{R},:)$ is bijective if and only if, for all $a, b \in R$ we have $a=b$ whenever $a p=b p$ for all $p \in B$.

Proposition 3.5. On a bijective Boolean module $\mathcal{M}=(\mathcal{B}, \mathcal{R},:)$ the pair $\left(\Delta_{B}, \theta_{2}\right)$ is a congruence on $\mathcal{M}$ if and only if $\theta_{2}=\Delta_{R}$.

Proof: If the cardinal of the set $R$ is 1 then $\Delta_{R}$ is the unique existing regular congruence. Let us admit that the cardinal of the set $R$ is great than 1 . If $\left(\Delta_{B}, \theta_{2}\right)$ is a congruence and if $\theta_{2} \neq \Delta_{R}$, then there exist distinct elements $a, b \in R$ such that $a \theta_{2} b$. Immediately, $a p \Delta_{B} b p$ for each $p \in B$, i.e., $a p=b p$ for every $p \in B$. Since $\mathcal{M}$ is bijective then $a=b$, a contradiction. Therefore $\theta_{2}=\Delta_{R}$.

Corollary 3.6. On a bijective Boolean module $\mathcal{M}=(\mathcal{B}, \mathcal{R},:)$ the pair $\left(\Delta_{B}, \nabla_{R}\right)$ is a congruence if and only if card $R=1$ (if and only if $\nabla_{R}=\Delta_{R}$ ). 
Adopting the general classic definition of a simple algebraic structure we are able to characterize the class of simple Boolean modules.

Definition 3.7. A Boolean module $\mathcal{M}=(\mathcal{B}, \mathcal{R},:)$ is simple whenever $\operatorname{Cong} \mathcal{M}=\left\{\left(\Delta_{B}, \Delta_{R}\right),\left(\nabla_{B}, \nabla_{R}\right)\right\}$.

Proposition 3.8. The degenerate Boolean module $\mathcal{M}=(\{0\},\{o\},:)$ is the unique simple Boolean module.

Proof: If the cardinal of the set $R$ is great than 1 then $R$ admits $\Delta_{R}$ and $\nabla_{R}$ as distinct congruences. Immediately $\left(\Delta_{B}, \Delta_{R}\right),\left(\nabla_{B}, \nabla_{R}\right)$ and $\left(\nabla_{B}, \Delta_{R}\right)$ are different congruences on $\mathcal{M}$ and, therefore, $\mathcal{M}$ is not a simple Boolean module.

If the cardinal of the set $R$ is 1 , we have $R=\{\mathrm{o}\}$ (with o $=e=1$ ). Then $B=\{0\}$. In fact, by $M_{4}$ and $M_{5}$ of Definition 2.4 we have $p=e p=\mathrm{o} p=0$ for every $p \in B$. Therefore $B=\{0\}$.

On a Boolean module $\mathcal{M}=(\mathcal{B}, \mathcal{R},:)$, Boolean congruences on $\mathcal{B}$ can exist that are not the Boolean part of any of its modular congruences. In fact, let $U=\{p, q\}$ and $\mathcal{M}$ be the full Boolean module over $U$. Since $I_{1}=\{\emptyset,\{p\}\}$ is a Boolean ideal on $\mathcal{B}(U)$, we can construct the Boolean congruence $\theta_{1}$ on $\mathcal{B}(U)$ defined by, for $s, r \in B,(s, r) \in \theta_{1}$ if and only if $s \vee i=r \vee i$, for some $i \in I_{1}$, with congruence classes $[0]_{\theta_{1}}=\{\emptyset,\{p\}\}$ and $[q]_{\theta_{1}}=\{\{q\}, U\}$. Let us admit the existence of a congruence $\theta_{2}$ on $\mathcal{R}(U)$ such that $\left(\theta_{1}, \theta_{2}\right)$ is a modular congruence on $\mathcal{M}$. Let $a \in \mathcal{R}(U)$ defined by $a=\{(q, p)\}$. We have $\emptyset \theta_{1}\{p\}$ and $a \theta_{2} a$, but $(a: \emptyset, a:\{p\}) \notin \theta_{1}$ (in fact, $a: \emptyset=\emptyset, a:\{p\}=\{q\}$ and $\left.(\emptyset,\{q\}) \notin \theta_{1}\right)$. Therefore, on the Boolean module $\mathcal{M}, \theta_{1} \in C$ ong $\mathcal{B}$ but it does not exist a congruence $\theta_{2} \in C o n g \mathcal{R}$ such that $\left(\theta_{1}, \theta_{2}\right) \in \operatorname{Cong} \mathcal{M}$.

Definition 3.9. Let $\mathcal{M}=(\mathcal{B}, \mathcal{R},:)$ be a Boolean module. A Boolean congruence $\theta_{1}$ on $\mathcal{B}$ is called pro-modular on $\mathcal{M}$ whenever there exists a congruence $\theta_{2}$ on $\mathcal{R}$ such that $\left(\theta_{1}, \theta_{2}\right)$ is a modular congruence on $\mathcal{M}$.

Proposition 3.10. Let $\mathcal{M}=(\mathcal{B}, \mathcal{R},:)$ be a Boolean module and let $\theta_{1}$ be a (Boolean) congruence on $\mathcal{B}$. The congruence $\theta_{1}$ is a pro-modular congruence on $\mathcal{M}$ if and only if the pair $\left(\theta_{1}, \Delta_{\mathcal{R}}\right)$ is a modular congruence on $\mathcal{M}$.

As previously done for dynamic algebras [7], next notion can also be established for Boolean modules.

Definition 3.11. Let $\mathcal{M}=(\mathcal{B}, \mathcal{R},:)$ be a Boolean module with the relation algebra $\mathcal{R}$ containing an element $\exists_{s}$ satisfying $\exists_{s} 0=0$ and $\exists_{s} p=1$ for every 
Boolean element $p \neq 0$. This element of $R$ is called the simple quantifier on $\mathcal{M}$.

Proposition 3.12. If $\mathcal{M}=(\mathcal{B}, \mathcal{R},:)$ is a Boolean module such that $\exists_{s} \in R$, then the congruences $\Delta_{B}$ and $\nabla_{B}$ are the only pro-modular ones.

Proof: Let $\theta_{1} \neq \Delta_{B}$ a pro-modular congruence on $\mathcal{M}$. There exists a Boolean element $p \neq 0$ such that $p \theta_{1} 0$. Since $\exists_{s} \in R$, then $\exists_{s} p \theta_{1} \exists_{s} 0$, so $1 \theta_{1} 0$. Therefore $\theta_{1}=\nabla_{B}$.

Corollary 3.13. If $\mathcal{M}=(\mathcal{B}, \mathcal{R},:)$ is a bijective Boolean module such that $\exists_{s} \in R$, then the congruences $\left(\Delta_{B}, \Delta_{R}\right)$ and $\left(\nabla_{B}, \theta_{2}\right)$ for every congruence $\theta_{2}$ on $\mathcal{R}$, are the only modular congruences on $\mathcal{M}$.

Proof: Using Propositions 3.12 and 3.5 we can infer that the congruence $\left(\Delta_{B}, \Delta_{R}\right)$ is the only modular congruence with $\Delta_{B}$ as Boolean part. We know that, for every congruence $\theta_{2}$ on $\mathcal{R}$, the pair $\left(\nabla_{B}, \theta_{2}\right)$ is a modular congruence on $\mathcal{M}$.

Corollary 3.14. For any set $U$, the congruences $\Delta_{B}$ and $\nabla_{B}$ are the only pro-modular congruences in the full Boolean module over $U, \mathcal{M}(U)=$ $(\mathcal{B}(U), \mathcal{R}(U),:)$.

Proof: The relation $\nabla_{R}$ is an element of $\mathcal{R}(U)$ and $\nabla_{R}$ is the simple quantifier on $\mathcal{M}(U)$.

Proposition 3.15. Let $\theta_{1}$ be a pro-modular congruence on a Boolean module $\mathcal{M}=(\mathcal{B}, \mathcal{R},:)$. Then

(1) $\left(\theta_{1}, \Delta_{\mathcal{R}}\right)$ is the smallest modular congruence on $\mathcal{M}$ having $\theta_{1}$ as Boolean part;

(2) $\phi=\left(\theta_{1},\{(a, b) \in R \times R\right.$ : there exists $j \in R$ such that $a \vee j=$ $b \vee j, j p \theta_{1} 0$ and $j p \theta_{1} 0$ for every $\left.\left.p \in B\right\}\right)$ is the greatest modular congruence on $\mathcal{M}$ having $\theta_{1}$ as Boolean part.

Proof: (1) Trivial.

(2) Our first aim is to show that $\phi=\left(\phi_{1}, \phi_{2}\right)$ with $\phi_{1}=\theta_{1}$ and $\phi_{2}=$ $\left\{(a, b) \in R \times R\right.$ : there exists $j \in R$ such that $a \vee j=b \vee j, j p \theta_{1} 0$ and $j p \theta_{1} 0$ for every $p \in B$ \} defines a congruence on $\mathcal{M}$.

(a) We prove that $\phi_{2}$ is an equivalence relation.

For $a \in R$, we have $a \vee \mathrm{o}=a \vee \mathrm{o}, \mathrm{o} p \theta_{1} 0$ and o $\breve{p} \theta_{1} 0$, and therefore $a \phi_{2} a$. 
Trivially if $a \phi_{2} b$ then $b \phi_{2} a$.

Let $a \phi_{2} b$ and $b \phi_{2} c$. So there exist $j, k \in R$ such that $a \vee j=$ $b \vee j, j p \theta_{1} 0$ and $j \breve{p} \theta_{1} 0$ for every $p \in B$ and $b \vee k=c \vee k, k p \theta_{1} 0$ and $k \breve{p} \theta_{1} 0$ for every $p \in B$.

But $a \vee(j \vee k)=(a \vee j) \vee k=(b \vee j) \vee k=(b \vee k) \vee j=(c \vee k) \vee j=$ $c \vee(j \vee k)$

Since $j p \theta_{1} 0$ and $k p \theta_{1} 0$ then $j p \vee k p \theta_{1} 0$ so $(j \vee k) p \theta_{1} 0$.

Since $j p \theta_{1} 0$ and $k \breve{k} \theta_{1} 0$ then $j \breve{p} \vee k \breve{p} \theta_{1} 0$ so $\left(j^{\sim} \vee k\right) p \theta_{1} 0$, i.e., $(j \vee k) \breve{p} \theta_{1} 0$

Therefore $a \phi_{2} c$.

(b) Let $a, b, c, d \in R$ such that $a \phi_{2} b$ and $c \phi_{2} d$. We have to prove that $a \breve{\phi_{2}} b,(a \wedge c) \phi_{2}(b \wedge d),(a \vee c) \phi_{2}(b \vee d)$ and $a c \phi_{2} b d$.

Since $a \phi_{2} b$ and $c \phi_{2} d$ there exist $j, k \in R$ such that $a \vee j=b \vee$ $j, j p \theta_{1} 0$ and $j \breve{p} \theta_{1} 0$ for every $p \in B$ and $c \vee k=d \vee k, k p \theta_{1} 0$ and $k \breve{p} \theta_{1} 0$ for every $p \in B$.

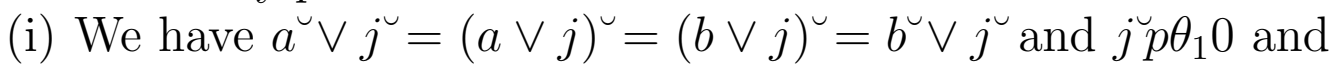
$j u p=j p \theta_{1} 0$ so $a \breve{\phi} b_{2} b$.

(ii) We have $(a \wedge c) \vee[(j \wedge c) \vee(a \wedge k) \vee(j \wedge k)]=(a \vee j) \wedge(c \vee k)=$ $(b \vee j) \wedge(d \vee k)=(b \wedge d) \vee[(j \wedge d) \vee(b \wedge k) \vee(j \wedge k)]$. Let $m=(j \wedge c) \vee(a \wedge k) \vee(j \wedge k)$ and $n=(j \wedge d) \vee(b \wedge k) \vee(j \wedge k)$.

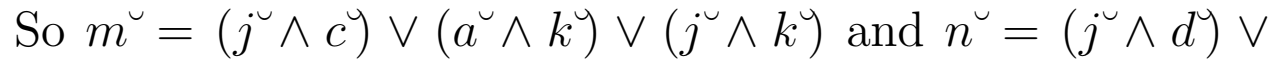
$\left(b^{\cup} \wedge k^{y}\right) \vee\left(j^{\cup} \wedge k^{y}\right)$. Since $(a \wedge c) \vee m=(b \wedge d) \vee n$ then $(a \wedge c) \vee(m \vee n)=(b \wedge d) \vee(m \vee n)$

Since for every $p \in B,(j \wedge c) p \leq j p,(a \wedge k) p \leq k p,(j \wedge k) p \leq$ $j p,(j \wedge d) p \leq j p,(b \wedge k) p \leq k p, j p \theta_{1} 0$ and $k p \theta_{1} 0$ then $(j \wedge c) p \theta_{1} 0,(a \wedge k) p \theta_{1} 0,(j \wedge k) p \theta_{1} 0,(j \wedge d) p \theta_{1} 0,(b \wedge k) p \theta_{1} 0$. So $m p \theta_{1} 0$ and $n p \theta_{1} 0$, and therefore $(m \vee n) p \theta_{1} 0$.

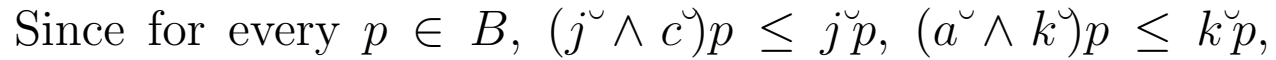

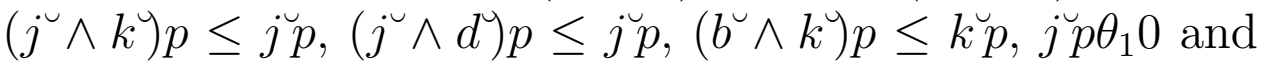
$k \breve{k} \theta_{1} 0$ then $\left(j \wedge c^{y}\right) p \theta_{1} 0,(a \wedge k) p \theta_{1} 0,(j \wedge k) p \theta_{1} 0,(j \wedge d) p \theta_{1} 0$,

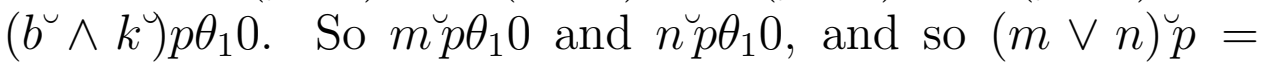
$(m \vee \vee n) p \theta_{1} 0$.

Therefore $(a \wedge c) \phi_{2}(b \wedge d)$.

(iii) We have $(a \vee c) \vee(j \vee k)=(b \vee d) \vee(j \vee k)$.

Since $j p \theta_{1} 0, k p \theta_{1} 0$ then $j p \vee k p \theta_{1} 0$, i.e., $(j \vee k) p \theta_{1} 0$.

Since $j \breve{p} \theta_{1} 0, k \breve{p} \theta_{1} 0$ then $j \breve{p} \vee k \breve{p} \theta_{1} 0$, i.e., $(j \vee k y) p \theta_{1} 0$. So $(j \vee k) \breve{p} \theta_{1} 0$. Therefore $(a \vee c) \phi_{2}(b \vee d)$. 
(iv) We have $a c \vee(a k \vee j d)=(a c \vee a k) \vee j d=a(c \vee k) \vee j d=$ $a(d \vee k) \vee j d=(a d \vee a k) \vee j d=(a \vee j) d \vee a k=(b \vee j) d \vee a k=$ $b d \vee(j d \vee a k)$. Let $l=a k \vee j d$. So $l^{\cup}=(a k) \vee(j d)^{\smile}=k \breve{a} \vee d j$. Since $k p \theta_{1} 0$ and $j p \theta_{1} 0$ for every $p \in B$ then $a k p \theta_{1} 0$ and $j d p \theta_{1} 0$ for every $p \in B$. So $(a k p \vee j d p) \theta_{1} 0$, i.e., $(a k \vee j d) p \theta_{1} 0$, i.e., $\operatorname{lp} \theta_{1} 0$. Since $k \breve{p} \theta_{1} 0$ and $j \breve{p} \theta_{1} 0$ for every $p \in B$ then

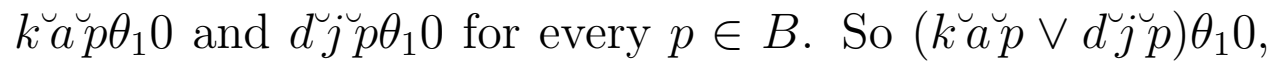
i.e., $\left(k \breve{a} \cup \vee d^{u}{ }^{\cup}\right) p \theta_{1} 0$, i.e., $l u p \theta_{1} 0$.

Therefore $a c \phi_{2} b d$.

(c) Let $p \theta_{1} q$ and $a \phi_{2} b$. We have to prove that $a p \theta_{1} b q$.

Since $a \phi_{2} b$ then there exists $j \in R$ such that $a \vee j=b \vee j, j p \theta_{1} 0$ and $j \breve{p} \theta_{1} 0$ for every $p \in B$.

So $(a \vee j) p=(b \vee j) p$, i.e., $a p \vee j p=b p \vee j p$. Since $j p \theta_{1} 0$ then $(a p \vee j p) \theta_{1}(0 \vee a p)$ and $(b p \vee j p) \theta_{1}(0 \vee b p)$, i.e., $(a p \vee j p) \theta_{1} a p$ and $(b p \vee j p) \theta_{1} b p$. So $a p \theta_{1} b p$. Since $p \theta_{1} q$ and $\theta_{1}$ is pro-modular then $b p \theta_{1} b q$. Therefore $a p \theta_{1} b q$.

(d) Now we have to prove that $\phi$ is the smallest modular congruence having $\theta_{1}$ as Boolean part, i.e., if $\theta=\left(\theta_{1}, \theta_{2}\right)$ is a modular congruence on $\mathcal{M}$, then $\theta \subseteq \phi$.

Let $a, b \in R$ and $a \theta_{2} b$. Since $\theta$ is a modular congruence on $\mathcal{M}$ we have $a^{\prime} \theta_{2} b^{\prime}$ and $a \theta_{2} b^{\lrcorner}$. So $\left(a^{\prime} \wedge b\right) \theta_{2} \mathrm{O}$ and $\left(a \wedge b^{\prime}\right) \theta_{2} \mathrm{O}$ and then $\left[\left(a^{\prime} \wedge b\right) \vee\left(a \wedge b^{\prime}\right)\right] \theta_{2}$ o. Therefore $(a \oplus b) \theta_{2} \mathrm{O}$ and $(a \oplus b) p \theta_{1} 0$.

We also have $\left(a^{\cup \prime} \wedge b^{\cup}\right) \theta_{2} \mathrm{O}$ and $\left(a^{\cup} \wedge b^{\lrcorner \prime}\right) \theta_{2} \mathrm{O}$ and then $\left[\left(a^{\cup \prime} \wedge b^{\cup}\right) \vee\left(a^{\cup} \wedge\right.\right.$

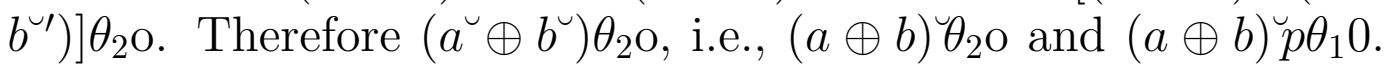
Since $a \vee(a \oplus b)=b \vee(a \oplus b)$ then $a \phi_{2} b$.

Definition 3.16. Let $\theta_{1}$ be a pro-modular congruence on a Boolean module $\mathcal{M}=(\mathcal{B}, \mathcal{R},:)$ and let $\phi=\left(\phi_{1}, \phi_{2}\right)$ be defined by

$\phi_{1}=\theta_{1}$

$\phi_{2}=\left\{(a, b) \in R \times R:\right.$ there exists $j \in R$ such that $a \vee j=b \vee j, j p \theta_{1} 0$ and $j \breve{p} \theta_{1} 0$ for every $\left.p \in B\right\}$.

The relation $\phi$ is called the determining congruence of any $\theta \in \operatorname{Cong} \mathcal{D}$ having $\theta_{1}$ as Boolean part (or simply a determining congruence).

Next example illustrates Proposition 3.15.

Example 3.17. Let $\mathcal{M}=(\mathcal{B}, \mathcal{R},:)$ be the proper Boolean module where $B=$ $\{\emptyset,\{p\},\{q\},\{p, q\}\}, R=\{\Lambda, a, b, c\}, \Lambda$ is the empty relation, $a=\{(p, p)\}$, 
$b=\{(q, q)\}$ and $c=\{(p, p),(q, q)\}$. Let $\theta_{1}$ be the Boolean congruence with congruence classes $[\emptyset]_{\theta_{1}}=\{\emptyset,\{p\}\}$ and $[\{p, q\}]_{\theta_{1}}=\{\{q\},\{p, q\}\}$, i.e.,

$\theta_{1}=\{(\emptyset, \emptyset),(\{p\},\{p\}),(\{q\},\{q\}),(\{p, q\},\{p, q\}),(\emptyset,\{p\}),(\{p\}, \emptyset),(\{q\},\{p, q\})$, $(\{p, q\},\{q\})\}$.

We have

$$
\begin{aligned}
& \Lambda^{\sim} \emptyset=\Lambda \emptyset=\emptyset \quad \square \backsim \emptyset=a \emptyset=\emptyset \\
& \Lambda^{\smile}\{p\}=\Lambda\{p\}=\emptyset \quad a^{\smile}\{p\}=a\{p\}=\{p\} \\
& \Lambda \smile\{q\}=\Lambda\{q\}=\emptyset \quad a\llcorner\{q\}=a\{q\}=\emptyset \\
& \Lambda \sim p, q\}=\Lambda\{p, q\}=\emptyset \quad a^{\cup}\{p, q\}=a\{p, q\}=\{p\} \\
& \vec{b} \emptyset=b \emptyset=\emptyset \quad c \breve{\emptyset}=c \emptyset=\emptyset
\end{aligned}
$$

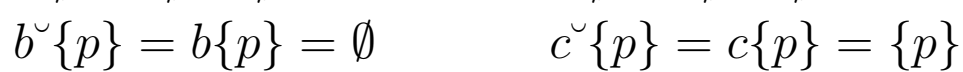

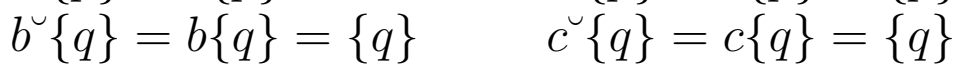

$$
\begin{aligned}
& b\{p, q\}=b\{p, q\}=\{q\} \quad c\llcorner\{p, q\}=c\{p, q\}=\{p, q\}
\end{aligned}
$$

So $\left(\theta_{1}, \Delta_{R}\right)$ is a modular congruence on $\mathcal{M}$ and then is the smallest (modular) congruence on $\mathcal{M}$ having $\theta_{1}$ as Boolean part.

The greatest modular congruence on $\mathcal{M}$ having $\theta_{1}$ as Boolean part is $\left(\theta_{1}, \phi_{2}\right)$ for $\phi_{2}=\{(f, g) \in R \times R$ : there exists $j \in R$ such that $f \vee j=$ $g \vee j, j s \theta_{1} 0$ and $j \breve{s} \theta_{1} 0$ for every $\left.s \in B\right\}$.

So $\Lambda$ and $a$ are the only elements $j$ of $R$ such that $j s \theta_{1} \emptyset$ and $j u s \theta_{1} \emptyset$ for every $s \in B$. Trivially we have $s \vee \Lambda=s \vee \Lambda$ for every $s \in R, \Lambda \vee a=a \vee a, b \vee a=c \vee a$ and for every $j \in\{\Lambda, a\}$ we have $\Lambda \vee j \neq b \vee j, \Lambda \vee j \neq c \vee j, a \vee j \neq b \vee j$ and $a \vee j \neq c \vee j$. So $\phi_{2}=\{(\Lambda, \Lambda),(a, a),(b, b),(c, c),(\Lambda, a),(a, \Lambda),(b, c),(c, b)\}$.

\section{The lattice $I d e \mathcal{M}$ of modular ideals}

Usually, the notion of ideal in a given class of algebras is established so that the zero-classes of congruence relations are easily seen to be ideals.

Definition 4.1. A (modular) ideal on a Boolean module $\mathcal{M}=(\mathcal{B}, \mathcal{R},:)$ is a pair $I=\left(I_{1}, I_{2}\right)$ satisfying the following conditions

(1) $I_{1}$ is a Boolean ideal on $\mathcal{B}$;

(2) If $p \in I_{1}$ and $a \in R$ then $a p \in I_{1}$;

(3) (a) $I_{2}$ is a Boolean ideal on $\mathcal{R}$;

(b) If $a \in I_{2}, c \in R$ then $a c, c a, a \cup \in I_{2}$;

(4) If $a \in I_{2}$ and $p \in B$, then $a p \in I_{1}$.

Such a subset $I_{2}$ of $R$ satisfying condition (3) is called an ideal of $R$. 
We denote by $I$ de $\mathcal{M}$ the set of all ideals on a Boolean module $\mathcal{M}=(\mathcal{B}, \mathcal{R},:)$. We intend to insert a lattice structure into $I d e \mathcal{M}$. To do so we need to define, for arbitrary modular ideals $I$ and $J, I \wedge_{\mathcal{M}} J$ and $I \vee_{\mathcal{M}} J$. It is immediate to put $I \wedge_{\mathcal{M}} J=\left(I_{1} \cap J_{1}, I_{2} \cap J_{2}\right)$. Once again the disjunction requires some attention. We denote by $\langle X\rangle_{\mathcal{A}}$ the ideal generated by a subset $X$ of any (homogeneous or heterogeneous) algebra $\mathcal{A}$, i.e., the intersection of all ideals $I$ on $\mathcal{A}$ containing $X$,

$$
\langle X\rangle_{\mathcal{A}}=\cap\{I: I \text { ideal on } \mathcal{A} \text { and } X \subseteq I\} .
$$

Proposition 4.2. Let $\mathcal{M}=(\mathcal{B}, \mathcal{R},:)$ a Boolean module, $I=\left(I_{1}, I_{2}\right)$ and $J=\left(J_{1}, J_{2}\right)$ be elements of Ide $\mathcal{M}$. We have

$\left\langle I_{1} \cup J_{1}\right\rangle_{\mathcal{B}}=\left\{p \in B: p \leq p_{1} \vee p_{2}\right.$, for some $\left.p_{i} \in I_{1} \cup J_{1}, i=1,2\right\}$

$\left\langle I_{2} \cup J_{2}\right\rangle_{\mathcal{R}}=\left\{a \in R: a \leq a_{1} \vee a_{2}\right.$, for some $\left.a_{i} \in I_{2} \cup J_{2}, i=1,2\right\}$.

Proof: We only have to prove the second identity since the first is a well known Boolean algebras result [4]. We want to show that, for $X=\{a \in R$ : $a \leq a_{1} \vee a_{2}$, for some $\left.a_{i} \in I_{2} \cup J_{2}, i=1,2\right\}$, we have

(i) $X$ is an ideal of $\mathcal{R}$;

(ii) $I_{2} \cup J_{2} \subseteq X$;

(iii) if $Y$ is an ideal of $\mathcal{R}$ and $I_{2} \cup J_{2} \subseteq Y$, then $X \subseteq Y$.

A well known Boolean algebras result states that $X$ is a Boolean ideal on $R$. Now, let $a \in X, b \in R$. Then $a \leq a_{1} \vee a_{2}$, for some $a_{i} \in I_{2} \cup J_{2}, i=1,2$. So

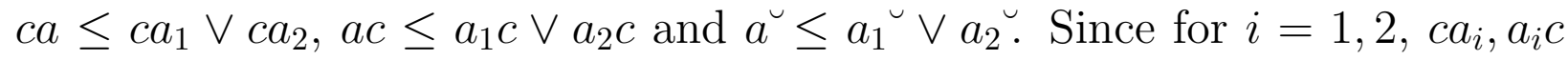

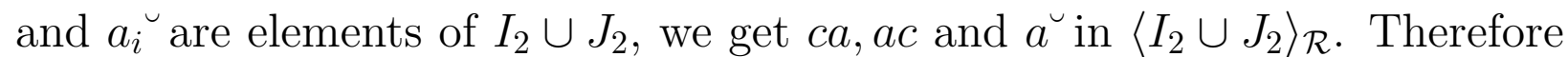
$X$ is an ideal of $\mathcal{R}$. It is straightforward that $I_{2} \cup J_{2} \subseteq X$. Let $a \in X$ and $Y$ be an ideal of $\mathcal{R}$ such that $I_{2} \cup J_{2} \subseteq Y$. Then $a \leq a_{1} \vee a_{2}$, for some $a_{i} \in I_{2} \cup J_{2} \subseteq Y, i=1,2$. But $Y$ is an ideal of $\mathcal{R}$ and $a_{i} \in Y$ for $i=1,2$ so $a_{1} \vee a_{2} \in Y$. Therefore $a \leq a_{1} \vee a_{2} \in Y$. Since $Y$ is an ideal of $\mathcal{R}$ we get $a \in Y$.

For $\mathcal{M}=(\mathcal{B}, \mathcal{R},:)$ Boolean module, $I \subseteq B$ and $J \subseteq R$ we write $J I$ to represent the set $J I=\{a p: a \in J$ and $p \in I\}$.

Proposition 4.3. Let $\mathcal{M}=(\mathcal{B}, \mathcal{R},:)$ be a Boolean module, $I=\left(I_{1}, I_{2}\right)$ and $J=\left(J_{1}, J_{2}\right)$ elements of Ide $\mathcal{M}$. We have

$$
\begin{aligned}
R\left(\left\langle I_{1} \cup J_{1}\right\rangle_{\mathcal{B}}\right) \subseteq\left\langle I_{1} \cup J_{1}\right\rangle_{\mathcal{B}}, \\
\left(\left\langle I_{2} \cup J_{2}\right\rangle_{\mathcal{R}}\right) B \subseteq\left\langle I_{1} \cup J_{1}\right\rangle_{\mathcal{B}} .
\end{aligned}
$$

Proof: Analogous to proposition on dynamic algebra [8]. 
Therefore the structure $\mathcal{I}$ de $\mathcal{M}=\left(\operatorname{Ide} \mathcal{M}, \wedge_{\mathcal{M}}, \vee_{\mathcal{M}}\right)$ where, for every $I=$ $\left(I_{1}, I_{2}\right), J=\left(J_{1}, J_{2}\right) \in I d e \mathcal{M}$, the operations are defined by

$$
\begin{aligned}
I \wedge_{\mathcal{M}} J & =I \cap J=\left(I_{1} \cap J_{1}, I_{2} \cap J_{2}\right) \\
I \vee_{\mathcal{M}} J & =\langle I \cup J\rangle_{\mathcal{M}}=\left(\left\langle I_{1} \cup J_{1}\right\rangle_{\mathcal{B}},\left\langle I_{2} \cup J_{2}\right\rangle_{\mathcal{R}}\right)
\end{aligned}
$$

is a lattice, called the lattice of ideals of $\mathcal{M}$.

Similarly to the congruences case, on a Boolean module $\mathcal{M}=(\mathcal{B}, \mathcal{R},:)$, Boolean ideals on $\mathcal{B}$ can exist that are not the Boolean part of any modular ideal on $\mathcal{M}$. In fact, let $U=\{p, q\}$ and $\mathcal{M}$ the full Boolean module over $U$. The set $I_{1}=\{\emptyset,\{p\}\}$ is a Boolean ideal on $\mathcal{B}(U)$ but, since for $a \in \mathcal{R}(U)$ given by $a=\{(q, p)\}$ we have $a:\{p\}=\{q\} \notin I_{1}$, the pair $\left(I_{1}, I_{2}\right)$ is not a modular ideal on $\mathcal{M}$, for any subset $I_{2}$ of $R$ (by 2 of Definition 4.1). Thus we are led to establish the following definition.

Definition 4.4. Let $\mathcal{M}=(\mathcal{B}, \mathcal{R},:)$ be a Boolean module. A Boolean ideal $I_{1}$ on $\mathcal{B}$ is called pro-modular on $\mathcal{M}$ if there exists an ideal $I_{2}$ of $\mathcal{R}$ such that $\left(I_{1}, I_{2}\right)$ is a modular ideal on $\mathcal{M}$.

Proposition 4.5. Let $\mathcal{M}=(\mathcal{B}, \mathcal{R},:)$ be a Boolean module and let $I_{1}$ be a Boolean ideal on $\mathcal{B}$. The ideal $I_{1}$ is a pro-modular ideal on $\mathcal{M}$ if and only if $\left(I_{1},\{o\}\right)$ is a modular ideal on $\mathcal{M}$.

Proposition 4.6. Let $I_{1}$ be a pro-modular ideal on a Boolean module $\mathcal{M}=$ $(\mathcal{B}, \mathcal{R},:)$. Then

(1) $\left(I_{1},\{o\}\right)$ is the smallest modular ideal having $I_{1}$ as Boolean part;

(2) $F=\left(I_{1},\left\{a:\right.\right.$ ap, $\breve{a p} \in I_{1}$ for every $\left.\left.p \in B\right\}\right)$ is the greatest modular ideal having $I_{1}$ as Boolean part.

Proof: (1) It is trivial that $\left(I_{1},\{0\}\right)$ is the smallest modular ideal having $I_{1}$ as Boolean part.

(2) Let $F=\left(I_{1}, F_{2}\right)$ with $F_{2}=\left\{a: a p, a \breve{p} \in I_{1}\right.$ for every $\left.p \in B\right\}$.

(a) $I_{1}$ is a Boolean ideal on $\mathcal{B}$;

(b) Since $I_{1}$ is pro-modular ideal, for $p \in I_{1}$ and $a \in R$ we have ap $\in I_{1}$

(c) (i) Since o $\in F_{2}(\stackrel{\circ}{p}=\mathrm{o} p=0$ for every $p \in B), F_{2} \neq \emptyset$. Let $a, b \in F_{2}$ and $d \in R$ such that $d \leq a$. So $a p, a \breve{p}, b p, b p \in$ $I_{1}$ for every $p \in B$. But $(a \vee b) p=a p \vee b p \in I_{1}$ and $(a \vee b) \breve{p}=(a \vee b \zeta) p=a \breve{p} \vee b \breve{p} \in I_{1}$, so $a \vee b \in F_{2}$. 
For every $p \in B$ we have $d p \leq a p \in I_{1}$, so $d p \in I_{1}$ and $d \breve{p} \leq a \breve{p} \in I_{1}$, so $d \breve{p} \in I_{1}$, and therefore $d \in F_{2}$.

So $F_{2}$ is a Boolean ideal on $\mathcal{R}$;

(ii) Let $a \in F_{2}$ and $c \in R$. So ap, $a \breve{p} \in I_{1}$ for every $p \in B$. Then

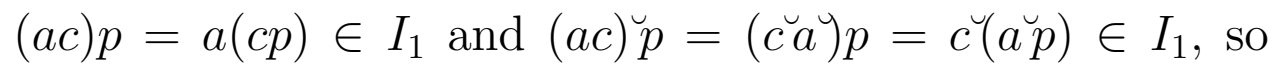

$$
\begin{aligned}
& a c \in F_{2} \text {. }
\end{aligned}
$$

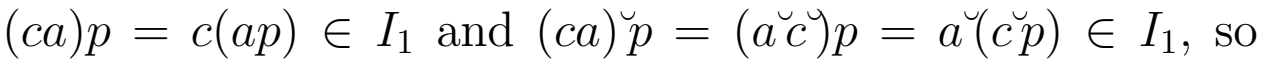

$$
\begin{aligned}
& c a \in F_{2} \text {. } \\
& a \breve{p} \in I_{1} \text { and } a \breve{p}=a p \in I_{1} \text {, so } a \breve{a} \in F_{2} \text {. }
\end{aligned}
$$

(d) By definition of $F_{2}$, if $a \in F_{2}$ and $p \in B$, then $a p \in I_{1}$. Therefore, $\left(I_{1}, F_{2}\right)$ is a modular ideal on $\mathcal{M}$.

Let $I=\left(I_{1}, I_{2}\right)$ be an arbitrary modular ideal on $\mathcal{M}$ and $a \in I_{2}$. Then $a \breve{u} \in I_{2}$ and $a p \in I_{1}$ for every $p \in B$. We also have $a \breve{p} \in I_{1}$ for every $p \in B$ establishing the conditions required to $a \in F_{2}$. Therefore, $\left(I_{1}, F_{2}\right)$ is the greatest modular ideal on $\mathcal{M}$ having $I_{1}$ as Boolean part.

Definition 4.7. Let $I_{1}$ be a pro-modular ideal on a Boolean module $\mathcal{M}=$ $(\mathcal{B}, \mathcal{R},:)$ and let $F=\left(F_{1}, F_{2}\right)$ be defined by

$F_{1}=I_{1}$

$F_{2}=\left\{a: a p, \breve{a p} \in I_{1}\right.$ for every $\left.p \in B\right\}$.

We say that $F$ is the determining ideal of any $I \in I d e \mathcal{M}$ having $I_{1}$ as Boolean part (or simply, a determining ideal).

Next example illustrates Proposition 4.6.

Example 4.8. Let $\mathcal{M}=(\mathcal{B}, \mathcal{R},:)$ be the Boolean module defined in Example 3.17, i.e., $B=\{\emptyset,\{p\},\{q\},\{p, q\}\}, R=\{\Lambda, a, b, c\}, \Lambda$ is the empty relation, $a=\{(p, p)\}, b=\{(q, q)\}$ and $c=\{(p, p),(q, q)\}$. Let $I_{1}$ be the Boolean ideal $I_{1}=\{\emptyset,\{p\}\}$.

We have

$$
\begin{array}{ll}
\Lambda^{\cup} \emptyset=\Lambda \emptyset=\emptyset & a^{\sim} \emptyset=a \emptyset=\emptyset \\
\Lambda^{\cup}\{p\}=\Lambda\{p\}=\emptyset & a^{\smile}\{p\}=a\{p\}=\{p\} \\
\Lambda^{\cup}\{q\}=\Lambda\{q\}=\emptyset & a^{\smile}\{q\}=a\{q\}=\emptyset \\
\Lambda^{\cup}\{p, q\}=\Lambda\{p, q\}=\emptyset & a^{\smile}\{p, q\}=a\{p, q\}=\{p\}
\end{array}
$$




$$
\begin{aligned}
& b \breve{\emptyset}=b \emptyset=\emptyset \quad c \breve{\emptyset}=c \emptyset=\emptyset \\
& b\{p\}=b\{p\}=\emptyset \quad c \backsim\{p\}=c\{p\}=\{p\}
\end{aligned}
$$

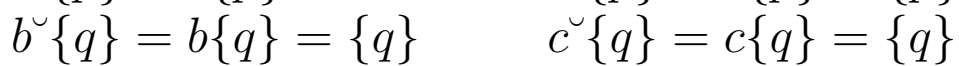

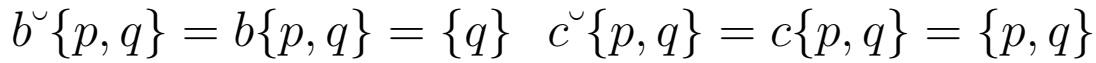

So $\left(I_{1},\{\Lambda\}\right)$ is a modular ideal on $\mathcal{M}$ and thus is the smallest ideal on $\mathcal{M}$ having $I_{1}$ as Boolean part.

The greatest modular ideal on $\mathcal{M}$ having $I_{1}$ as Boolean part is $\left(I_{1}, F_{2}\right)$ with $F_{2}=\left\{f \in R: f s, f^{\sim} s \in I_{1}\right.$ for every $\left.s \in B\right\}$ and $\Lambda$ and $a$ are the only elements $j$ of $R$ such that $j s, j s \in I_{1}$ for every $s \in B$. Therefore $F_{2}=\{\Lambda, a\}$.

\section{Modular Congruences and Modular Ideals}

The main purpose of this paragraph is to establish that the class of Boolean module is ideal determined [5], i.e., that each modular ideal is the zero-class of a unique modular congruence.

Definition 5.1. If $\theta=\left(\theta_{1}, \theta_{2}\right) \in \operatorname{Cong} \mathcal{M}$ where $\mathcal{M}=(\mathcal{B}, \mathcal{R},:)$ is a Boolean module, we say that $\mathcal{I}(\theta)=\mathcal{I}^{\theta}=\left(\mathcal{I}_{1}^{\theta}, \mathcal{I}_{2}^{\theta}\right)$ defined by

$$
\begin{aligned}
& \mathcal{I}_{1}^{\theta}=\left\{p \in B: p \theta_{1} 0\right\}=[0]_{\theta_{1}} \\
& \mathcal{I}_{2}^{\theta}=\left\{a \in R: a \theta_{2} \mathrm{O}\right\}=[\mathrm{o}]_{\theta_{2}}
\end{aligned}
$$

is the kernel of the congruence $\theta$.

Proposition 5.2. The kernel $\mathcal{I}(\theta)$ of a congruence $\theta$ on a Boolean module $\mathcal{M}=(\mathcal{B}, \mathcal{R},:)$ is an ideal on $\mathcal{M}$.

Proof: (1) The fact that $\mathcal{I}_{1}^{\theta}$ is a Boolean ideal on $\mathcal{B}$ is a known Boolean algebras result.

(2) We have to prove that if $p \in \mathcal{I}_{1}^{\theta}$ and $a \in R$ then $a p \in \mathcal{I}_{1}^{\theta}$. In fact, if $a \in R$ and $p \in \mathcal{I}_{1}^{\theta}$, then $p \theta_{1} 0$ and $a \theta_{2} a$. Therefore $(a p) \theta_{1}(a 0)$, i.e., (ap) $\theta_{1} 0$. So $a p \in \mathcal{I}_{1}^{\theta}$.

(3) (a) The fact that $\mathcal{I}_{2}^{\theta}$ is a Boolean ideal on $\mathcal{R}$ is again a known Boolean algebras result.

(b) We have to prove that, if $a \in \mathcal{I}_{2}^{\theta}$ and $c \in R$, then $a c, c a, a^{\sim} \in$ $\mathcal{I}_{2}^{\theta}$. In fact, since $a \in \mathcal{I}_{2}^{\theta}$ then $a \theta_{2} \mathrm{O}$, and therefore, since $c \theta_{2} c$ then $(a c) \theta_{2}(\mathrm{oc})$ and $(c a) \theta_{2}(c \mathrm{o})$, i.e., $(a c) \theta_{2} \mathrm{O}$ and $(c a) \theta_{2} \mathrm{O}$. To this extend $a c$ and $c a$ are elements of $\mathcal{I}_{2}^{\theta}$. Since $a \theta_{2} \mathrm{O}$ and $\theta_{2}$ is a

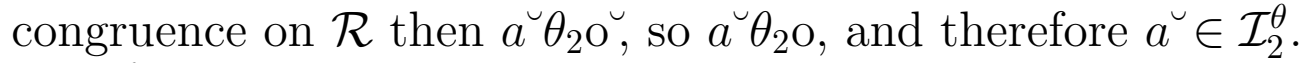

(4) Let $a \in \mathcal{I}_{2}^{\theta}$ and $p \in B$. Then $a \theta_{2} \mathrm{O}$ and $p \theta_{1} p$, and therefore $(a p) \theta_{1} \mathrm{o} p$, i.e., (ap) $\theta_{1} 0$. Immediately ap $\in \mathcal{I}_{1}^{\theta}$. 
Definition 5.3. The kernel of a Boolean modular homomorphism $h=$ $\left(h_{1}, h_{2}\right): \mathcal{M} \longrightarrow \mathcal{M}^{\prime}$ between Boolean modules is the pair $\left(\left\{p \in B: h_{1}(p)=\right.\right.$ $\left.0\},\left\{a \in R: h_{2}(a)=\mathrm{o}\right\}\right)$.

Proposition 5.4. The kernel of a Boolean modular homomorphism $h: \mathcal{M} \longrightarrow \mathcal{M}^{\prime}$ between Boolean modules is a modular ideal on $\mathcal{M}$.

Proof: Trivial.

Definition 5.5. If $I=\left(I_{1}, I_{2}\right)$ is a modular ideal on a Boolean module $\mathcal{M}=(\mathcal{B}, \mathcal{R},:)$, we define $\mathcal{C}(I)=\mathcal{C}^{I}=\left(\mathcal{C}_{1}^{I}, \mathcal{C}_{2}^{I}\right)$ by

$p \mathcal{C}_{1}^{I} q$ if and only if $p \vee i=q \vee i$ for some $i \in I_{1}$,

$a \mathcal{C}_{2}^{I} b$ if and only if $a \vee j=b \vee j$ for some $j \in I_{2}$, for $p, q \in B$ and $a, b \in R$.

Proposition 5.6. If $I=\left(I_{1}, I_{2}\right)$ is a modular ideal on a Boolean module $\mathcal{M}=(\mathcal{B}, \mathcal{R},:)$, then $\mathcal{C}(I)$ is a modular congruence on $\mathcal{M}$.

Proof: (i) The relation $\mathcal{C}_{1}^{I}$ is a congruence relation on $\mathcal{B}$, a known result in Boolean algebras.

(ii) The relation $\mathcal{C}_{2}^{I}$ is a Boolean congruence relation on $\mathcal{R}$, a known result in Boolean algebras. To prove that the relation $\mathcal{C}_{2}^{I}$ is a congruence relation on $\mathcal{R}$ we have to prove that, for $a, b, c, d \in R$, if $a \mathcal{C}_{2}^{I} b$ and $c \mathcal{C}_{2}^{I} d$ then $(a c) \mathcal{C}_{2}^{I}(b d)$ and $\left(a^{y}\right) \mathcal{C}_{2}^{I}\left(b^{y}\right)$. Let us admit that $a \mathcal{C}_{2}^{I} b$ and $c \mathcal{C}_{2}^{I} d$. Then there exist $j, k$ in $I_{2}$ such that $a \vee j=b \vee j$ and $c \vee k=d \vee k$. Now

(a) From $c \vee k=d \vee k$ we get $a(c \vee k)=a(d \vee k)$, i.e., $a c \vee a k=a d \vee a k$. Hence $a c \vee(a k \vee j d)=a(c \vee k) \vee j d=a(d \vee k) \vee j d=(a d \vee a k) \vee j d=$ $(a \vee j) d \vee a k=(b \vee j) d \vee a k=b d \vee(j d \vee a k)$. Since $j d \vee a k \in I_{2}$ we get $(a c) \mathcal{C}_{2}^{I}(b d)$.

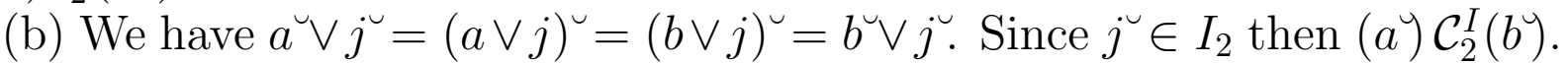

(iii) Now we have to prove that, for $a, b \in R$ and $p, q \in B$, if $a \mathcal{C}_{2}^{I} b$ and $p \mathcal{C}_{1}^{I} q$ then $(a p) \mathcal{C}_{1}^{I}(b q)$. Since $p \mathcal{C}_{1}^{I} q$ and $a \mathcal{C}_{2}^{I} b$, then $p \vee i=q \vee i$ for some $i \in I_{1}$ and $a \vee j=b \vee j$ for some $j \in I_{2}$ (and therefore $a q \vee j q=b q \vee j q$ ). But from $p \vee i=q \vee i$ we get $a p \vee a i=a q \vee a i$ and moreover $a p \vee a i \vee j q=a q \vee a i \vee j q$. So $a p \vee(a i \vee j q)=(a p \vee a i) \vee j q=(a q \vee a i) \vee j q=(a q \vee j q) \vee a i=(b q \vee j q) \vee a i=$ $b q \vee(a i \vee j q)$. Since $a i \vee j q \in I_{1}$ then $(a p) \mathcal{C}_{1}^{I}(b q)$.

Definition 5.7. For $I$ a modular ideal on a Boolean module, we say that $\mathcal{C}(I)$ is the congruence induced by $I$.

Proposition 5.8. If $I=\left(I_{1}, I_{2}\right)$ is a modular ideal on a Boolean module, then $\mathcal{I}(\mathcal{C}(I))=I$. 
Proof: Similar to Boolean algebras.

Proposition 5.9. On a Boolean module a modular ideal is a determining ideal if and only if it is the kernel of a determining congruence.

Proof: Let $I=\left(I_{1}, I_{2}\right)$ be a modular ideal on a Boolean module $\mathcal{M}=$ $(\mathcal{B}, \mathcal{R},:)$. By Prop.5.8 there exists a modular congruence $\theta$ such that $I=$ $\mathcal{I}(\theta)$. Let $\phi$ be the determining congruence of $\theta$. We have $\phi_{1}=\theta_{1}$ and $\phi_{2}=$ $\left\{(a, b) \in R \times R\right.$ : there exists $j \in R$ such that $a \vee j=b \vee j, j p \theta_{1} 0$ and $j \breve{p} \theta_{1} 0$ for every $p \in B\}$. So, $\mathcal{I}_{1}^{\phi}=\mathcal{I}_{1}^{\theta}=I_{1}$ and $\mathcal{I}_{2}^{\phi}=\left\{a: a \phi_{2} \mathrm{O}\right\}$. Then, we have to prove that $\left\{a: a \phi_{2} \mathrm{O}\right\}=\left\{a: a p, a \breve{p} \in I_{1}\right.$ for every $\left.p \in B\right\}$.

Let $a_{1} \in\left\{a: a \phi_{2} \mathrm{O}\right\}$. There exists $j \in R$ such that $a_{1} \vee j=\mathrm{o} \vee j, j p \theta_{1} 0$ and $j p \theta_{1} 0$ for every $p \in B$. Since $a_{1} \vee j=o \vee j=j$ then $a_{1} p \vee j p=j p$ and

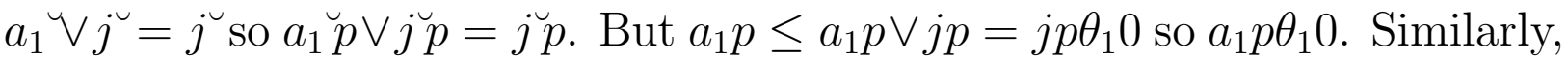
we have $a_{1} \breve{p} \leq a_{1} \breve{p} \vee j \breve{p}=j \breve{p} \theta_{1} 0$ so $a_{1} \breve{p} \theta_{1} 0$ and then $a_{1} p, a_{1} \breve{p} \in[0]_{\theta_{1}}=I_{1}$.

Let $a_{1} \in\left\{a: a p, a \breve{p} \in I_{1}\right.$ for every $\left.p \in B\right\}$. We have to prove that $a_{1} \phi_{2} \mathrm{O}$, i.e., there exists $j \in R$ such that $a_{1} \vee j=j, j p \theta_{1} 0$ and $j \breve{p} \theta_{1} 0$ for every $p \in B$. Since $a_{1} p, a_{1} \breve{p} \in I_{1}$ for every $p \in B$ and $I_{1}=[0]_{\theta_{1}}$ then $a_{1} p \theta_{1} 0$ and $a_{1} \breve{p} \theta_{1} 0$. Since $a_{1} \vee a_{1}=a_{1}$ putting $j=a_{1}$ we have the required.

Proposition 5.10. If $\theta=\left(\theta_{1}, \theta_{2}\right)$ is a congruence on a Boolean module, then $\mathcal{C}(\mathcal{I}(\theta))=\theta$.

Proof: As in Boolean algebras.

Proposition 5.11. On a Boolean module a modular congruence is a determining congruence if and only if is the congruence induced by a determining ideal.

Proof: If $\phi$ is a determining congruence on a Boolean module, Proposition 5.9 asserts that $\mathcal{I}(\phi)=F$ for some determining ideal $F$. So $\mathcal{C}(\mathcal{I}(\phi))=\mathcal{C}(F)$. But Proposition 5.10 infers that $\phi=\mathcal{C}(F)$.

If $F$ is a determining ideal on a Boolean module, using Proposition 5.9 we have $\mathcal{I}(\phi)=F$ for some determining congruence $\phi$. So $\mathcal{C}(\mathcal{I}(\phi))=\mathcal{C}(F)$. By Proposition 5.10 we have $\phi=\mathcal{C}(F)$ as required.

Theorem 5.12. The pair of maps $\mathcal{C}:$ Ide $\mathcal{M} \longrightarrow \operatorname{Cong} \mathcal{M}$ (that for each $I \in I$ de $\mathcal{M}$ assigns the congruence $\mathcal{C}(I)$ ) and $\mathcal{I}: \operatorname{Cong} \mathcal{M} \longrightarrow$ Ide $\mathcal{M}$ (that for each $\theta \in C$ Cong $\mathcal{M}$ assigns the ideal $\mathcal{I}(\theta)$ ) defines an isomorphism between the lattices Ide $\mathcal{M}$ and $\operatorname{Cong} \mathcal{M}$. 
Proof: As in Boolean algebras.

We infer that the class of the Boolean module is ideal determined, i.e., each ideal is the zero-class of a unique congruence. We can easily affirm that the modular ideal $F$ defined on Proposition 4.6 is the kernel of the modular congruence $\phi$ presented on Proposition 3.15. Conversely, the congruence $\phi$ defined on Proposition 3.15 is the congruence induced by the modular ideal $F$ constructed on Proposition 4.6, i.e., either Proposition 4.6 and Proposition 3.15 can now be stated as corollaries of each other using Theorem 5.12.

\section{References}

[1] C. Brink. Boolean Modules, Journal of Algebra 71, 291-313 (1981).

[2] C. Brink, K. Britz, and R. Schmidt. Peirce Algebras, Formal Aspects of Computing 6, 339-358 (1994).

[3] L. H. Chin, and A. Tarski. Distributive and modular laws in the arithmetic of relation algebras, University of California Publications in Mathematics 1, 341-384 (1951).

[4] G. Gratzer. General Lattice Theory, (Birkhauser Verlag, 1998).

[5] H.P. Gumm, and A. Ursini. Ideals in universal algebras, Algebra Universalis 19, 45-54 (1984).

[6] R. Hirsch. Peirce algebras and Boolean modules, J Logic Computation 17, 255-283 (2007).

[7] S. M. Pinto, M.T. Oliveira-Martins, and M.C. Pinto. Monadic Dynamic Algebras, Math. Log. Quart. 52, 134-150 (2006).

[8] S. M. Pinto, M.T. Oliveira-Martins, and M.C. Pinto. Congruences on Dynamic Algebras, Logic J. IGPL 16, 15-31 (2008).

[9] A. Tarski. On the calculus of relations, J. Symbolic Logic 6, 73-89 (1941).

[10] A. Tarski. Contributions to the theory of models, Part II, Indagationes Mathematical 17, 56-64 (1955).

Sandra Marques Pinto

Departamento de Matemática, Universidade de Coimbra, 3001-454 Coimbra, PORTUGal

E-mail address: sandra@mat.uc.pt

M. Teresa Oliveira-Martins

Departamento de Matemática, Universidade de Coimbra, 3001-454 Coimbra, POrTugal

E-mail address: meresa@mat.uc.pt 